\title{
Helicobacter pylori infection related long noncoding RNA (IncRNA) AF147447 inhibits gastric cancer proliferation and invasion by targeting MUC2 and up-regulating miR-34c
}

\author{
Xiaoying Zhou ${ }^{1,2, *}$, Han Chen ${ }^{1,2, *}$, Li Zhu ${ }^{3, *}$, Bo Hao ${ }^{1}$, Weifeng Zhang ${ }^{1}$, Jie Hua ${ }^{1}$, \\ Huiyuan $\mathbf{G u}^{4}$, Wujuan Jin ${ }^{1,2}$, Guoxin Zhang ${ }^{1,2}$ \\ ${ }^{1}$ Department of Gastroenterology, First Affiliated Hospital of Nanjing Medical University, Nanjing, China \\ ${ }^{2}$ First Clinical Medical College of Nanjing Medical University, Nanjing, China \\ ${ }^{3}$ Department of Gastroenterology, People's Hospital of Jingjiang, Taizhou, China \\ ${ }^{4}$ Department of Gastroenterology, First Affiliated Hospital of Soochow University, Suzhou, China \\ "These authors contributed equally to this work
}

Correspondence to: Guoxin Zhang, email: guoxinz@njmu.edu.cn

Keywords: helicobacter pylori infection, IncRNA, gastric cancer, MUC2

Received: April 27, $2016 \quad$ Accepted: October 10, $2016 \quad$ Published: November 07, 2016

\section{ABSTRACT}

Long non-coding RNAs (IncRNAs) were shown to play critical roles in cancer biology. We investigated whether $H$. pylori infection could promote gastric cancer by regulating IncRNAs expression. Differentially expressed IncRNAs between $\boldsymbol{H}$. pylori positive and negative tissues were identified by microarray and validated by qRT-PCR. Our results indicated that $\boldsymbol{H}$. pylori positive tissues have a specific profile of IncRNAs. Cell biological assays with siRNA-mediated knockdown or lentivirus vector-mediated over-expression were performed to probe the functional relevance of the IncRNAs. We identified an IncRNA-AF147447 decreased expressed by $H$. pylori infection, which can inhibit GC proliferation and invasion in vitro and in vivo, act as a tumor suppressor in the development of $H$. pylori induced GC. LncRNA AF147447 could repress MUC2 expression by direct binding or increasing miR-34c expression. We also found that transcription factor E2F1 could be recruited to IncRNA AF147447 promoter by RNA immunoprecipatation and RNA pull down assays. These findings support a role of IncRNA AF147447 in tumor suppression. This discovery contributes to a better understanding of the importance of the deregulated IncRNAs by $\boldsymbol{H}$. pylori infection and provides a rationale for the potential development of IncRNA-based targeted approaches for the treatment of $\boldsymbol{H}$. pylori-related gastric cancer.

\section{INTRODUCTION}

Gastric cancer (GC) belongs to the third leading cause of cancer-related death in the worldwide [1]. A lot of risk factors contribute to $\mathrm{GC}$, among which Helicobacter pylori (H. pylori) is the strongest one [2]. The rate of $H$. pylori infection is over $50 \%$ worldwide nowadays, especially among developing countries [3]. The mechanism considering how $H$. pylori infection induces GC has not been fully identified [4].

Long non-coding RNAs (LncRNAs) are a group of non-coding RNA transcripts which are longer than 200 nucleotides (nt) [5]. Normally, they have little or no protein capacity. Like miRNAs, IncRNAs play important roles in regulating target gene expression, by transcription, posttranscriptional processing or epigenetically regulation, including binding with miRNA or chromatin modification and genomic imprinting [6], and therefore, IncRNAs are associated with various diseases, such as metabolic disorders [7], cardiac diseases [8], tumors [9], etc.

Numerous studies have suggested the role of dysregulated lncRNA expression in GC. Zhang et al [10] found that IncRNA ANRIL was significantly upregulated in $\mathrm{GC}$ tissues. By recruiting and binding to PRC2, ANRIL epigenetically repress miR-99a/miR-449a, like ceRNAs, in controlling the targets-mTOR and CDK6/E2F1 pathway. $\mathrm{Xu}$ et al [11] found that lncRNA FENDRR expression was down-regulated in both GC cell lines and tissues 
and it could be considered as diagnostic and prognostic markers for GC patients. However, until now, no studies were performed regarding the dysregulated lncRNAs in $H$. pylori infection-related GC.

In this study, we were the first to explore the dysregulated IncRNA expression in $H$. pylori positive tissues by IncRNA microarray. Our study showed that numbers of IncRNAs were dysregulated. We further investigated the detailed function of a $H$. pylori infectionrelated down-regulated IncRNA, AF147447, in vivo and in vitro, and found that it plays an important role in H. pylori-related GC.

\section{RESULTS}

\section{Differential LncRNA Expressions were found in the gastric tissues of $\mathrm{H}$. pylori positive and negative patients}

We chose 3 matching pairs of $H$. pylori infected and non-infected gastric tissues for microarray analysis of lncRNAs and mRNAs. We set a threshold as a fold change that is larger than 2.0 and a $p$ value that is smaller than 0.05 and found that there were 123 dysregulated lncRNAs and 87 dysregulated mRNAs in the gastric tissues of H. pylori infected and non-infected patients, which means that the lncRNA (Supplementary Figure S1A) and mRNA (Supplementary Figure S1B) expression level were significantly different between the two groups. In order to validate microarray analysis findings, top ten lncRNAs from the differentially expressed lncRNAs were randomly selected with a fold change that is larger than 5 and detected their expressions by real time (RT)-PCR. The PCR validation data were the same as the microarray results (Supplementary Figure $\mathrm{S} 1 \mathrm{C}, p<0.05$ for all). Consequently, our results suggested that a set of lncRNAs were frequently dysregulated in the gastric tissues of H. pylori infected and control patients and they may be related to the $H$. pylori-related carcinogenesis.

\section{LncRNA AF147447 was decreased expressed in $H$. pylori infected tissues and cells}

Among the lncRNAs differentially expressed in tissues, we found that an lncRNA, accession number AF147447 in NCBI, was significantly decreased by $H$. pylori infection. Its expression decreased most significantly in qRT-PCR analysis (Supplementary Figure S1C). Consequently, we chose this lncRNA for further functional analysis and investigate potential mechanisms underlying H. pylori induced GC. In order to analyze in vitro whether H. pylori infection alters AF147447 expression, we measured AF147447 expression after H. pylori co-culture with GC cells. We found that AF147447 expression was decreased dose-dependently with $H$. pylori infection MOIs in three gastric epithelial cells (Figure 1A).
Then, we detected AF147447 expression in 75 pairs of H. pylori infected and non-infected tissues and 50 pairs of tumor tissues by qRT-PCR. The basic characteristics of enrolled patients were shown (Supplementary Table S1) and the results showed that AF147447 was significantly decreased when $H$. pylori infected, regardless of control or tumor tissues. Additionally, we also found out that the expression of AF147447 was significantly decreased in tumor tissues (Figure 1B). When compared its expression with clinical characteristics, we found that patients with intestinal metaplasia expressed significantly lower AF147447 compared with superficial gastritis or atrophic gastritis (Figure 1C), which suggested that the more severe the histology of the patients is, the lower AF147447 expressed. In 50 pairs of tumor tissues, we found that patients with advanced stages showed significantly lower AF147447 expression (Supplementary Figure S2). We next examined AF147447 expression in $H$. pylori infected gastric tissues in mice. Infected mice lasted for one year yielded the lowest AF147447 expression (Figure 1F), which showed that AF147447 expression was decreased both time dependently and dose dependently with $H$. pylori infection.

\section{Cell proliferation and cell migration were inhibited by IncRNA AF147447 in vitro and in vivo}

LncRNA AF147447 down-regulation in H. pylori positive tissues suggested that it may play an important role in $H$. pylori linked carcinogenesis process. Thus, we investigated cell proliferation, migration, and invasion by decreasing or increasing AF147447 expression. Firstly, we examined the expression of AF147447 in 5 gastric epithelial cells. We found that AF147447 expression was lowest in 7901 and highest in MKN45 (Supplementary Figure S1C). Consequently, we chose 7901 to over-express AF147447 and MKN45 to reduce AF147447 expression. 3 siRNAs were used to interfere with AF147447 expression and siRNA2 showed the highest inhibition rate (Figure 2A). We chose siRNA2 for further functional analysis. From CCK8 analysis, we found that suppression of AF147447 greatly enhanced cell proliferation (Figure 2B). Also, AF147447 inhibition also promoted GC cells invasion activity compared with controls (Figure 2C). We also found in vivo that comparing to those injected with siRNA controls, mice injected with AF147447 siRNA exhibited significantly larger tumor volume and faster tumor growth (Figure 2D). We then extracted RNA from the tumors and found that AF147447 expression was significantly lower after siRNA injection by qRT-PCR analysis.

Next, we over-expressed AF147447 by pcDNA or lentivirus. We found that after pcDNA transfection or lentivirus infection, the expression of AF147447 was significantly up-regulated (Figure 3A). From CCK8 
analysis, we found that over-expression of AF147447 significantly inhibit cell proliferation (Figure 3B). It also inhibited GC cells invasion activity after pcDNA transfection or lentivirus infection compared with controls (Figure 3C). For nude mice xenoplantation, GC cells transfected with pLV-AF147447 or control were injected. Mice which were injected with pLV-AF147447 exhibited much lower tumor growth and less tumor volume compared with mice injected with controls (Figure 3D). AF147447 expression from the tumors was significantly higher in $\mathrm{pLV}$ AF147447 transfected mice by qRT-PCR analysis. Together, these results showed that, both in vitro and in vivo, lncRNA AF147447 inhibited cell proliferation and migration.

\section{LncRNA AF147447 could bind with and target oncogene MUC2}

Studies have been suggested that IncRNAs play their regulatory roles in inducing tumorigenesis mainly by binding with their target gene products. Consequently, in order to figure out whether this lncRNA could function by this way, RNA pull-down analysis was therefore performed to identify all the possible proteins that were bind with AF147447 (Figure 4A). MUC2 was therefore, revealed by this mass spectrometry analysis, specifically associated with AF147447 among one of the many binding proteins (Supplementary Table S2).

Previous studies showed that MUC2 overexpression was related to $\mathrm{GC}$ progression and poor prognosis. In order to validate the relationship between AF147447 and MUC2, luciferase reporter assay was performed with MUC2 promoter region. LncRNA AF147447 could combine with MUC2 promoter region through sequence match (Figure 4B). The target sequence of the mutant (mt) or wild type (wt) promoter was cloned into a luciferase reporter vector. We then transfected the cells with wt/ mt vector together with lncRNA AF147447. We found that cells transfected with wt promoter presented with a marked decrease of luciferase activity, comparing with those transfected with NC (Figure 4B, $p<0.01$ ). However, the luciferase activity of $\mathrm{mt}$ vector remained the same after a simultaneous transfection with AF147447.

Then, the functional relevance between lncRNA AF147447 and MUC2 was determined. LncRNA
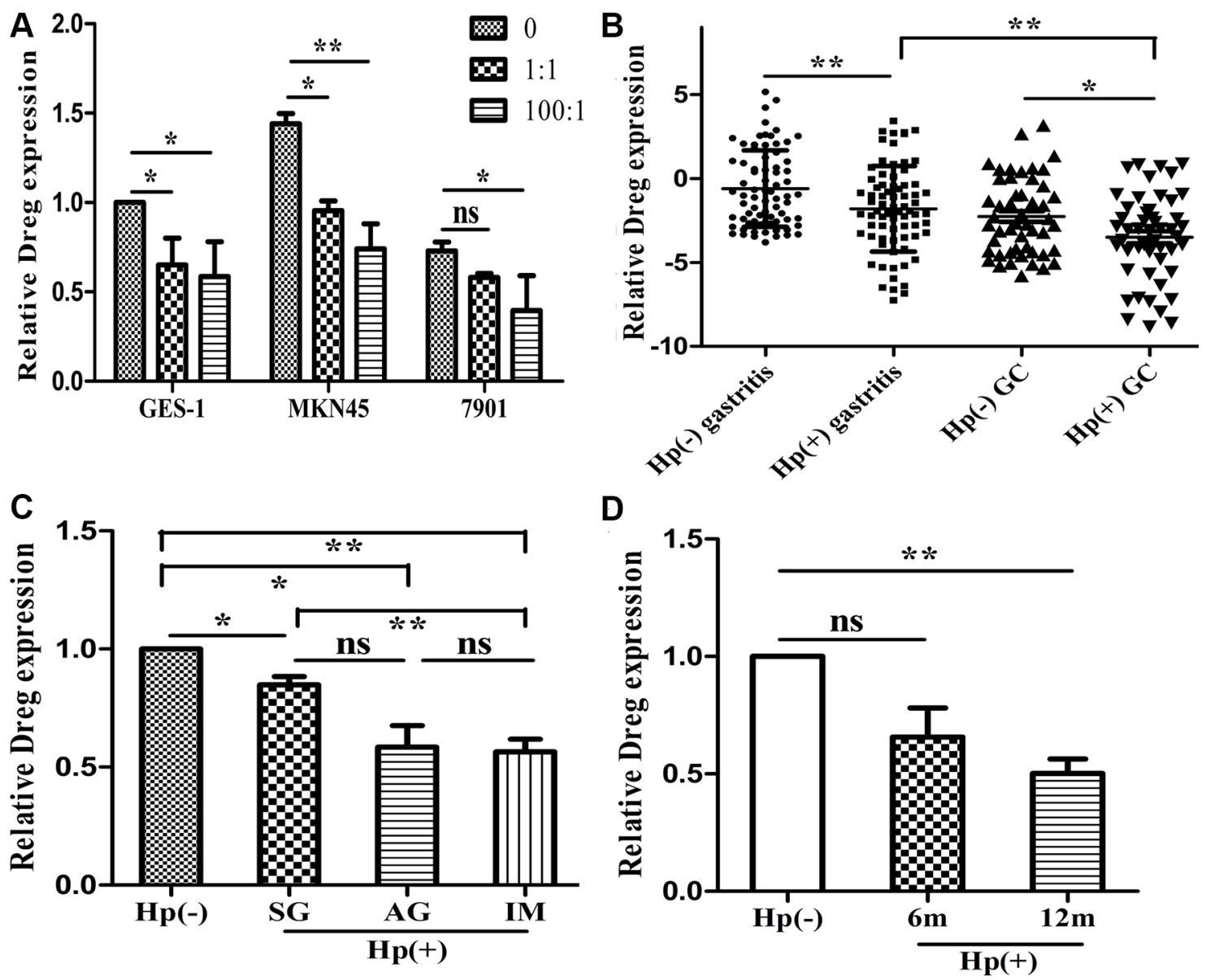

Figure 1: LncRNA AF147447 expression in $\boldsymbol{H}$. pylori infected state. (A) AF147447 expression in $H$. pylori infected cells compared with controls. (B) AF147447 expression in H. pylori infected gastritis and GC tissues compared with their respective controls. (C) AF147447 expression in different histology type of $H$. pylori infected gastritis. (D) AF147447 expression in H. pylori infected gastric tissues in mice. $\left({ }^{*} p<0.05,{ }^{*} p<0.01\right)$. 
AF147447 could inhibit or promote cell proliferation and invasion through targeting and changing the expression of MUC2. In order to test this hypothesis, we measured the RNA (Figure 4C) and protein (Figure 4D) expression levels of MUC2 by qRT-PCR and western blot in 7901 cells with AF147447 overexpression or decreased expression. We found that MUC2 expression was markedly decreased in the cells stably transfected with pLV-AF147447 than that with pLV-NC, and was significantly increased transfecting with AF147447 siRNA
A

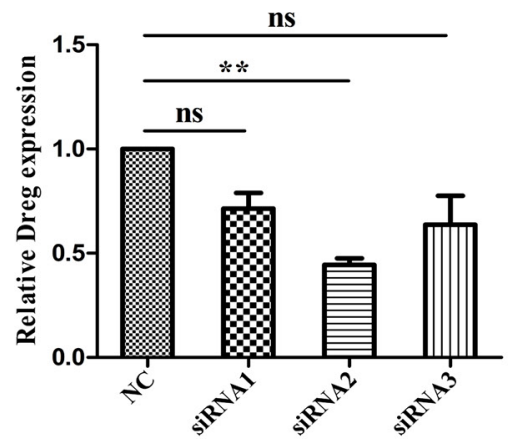

C
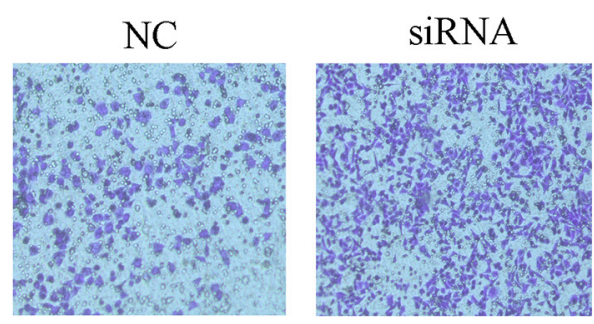

D

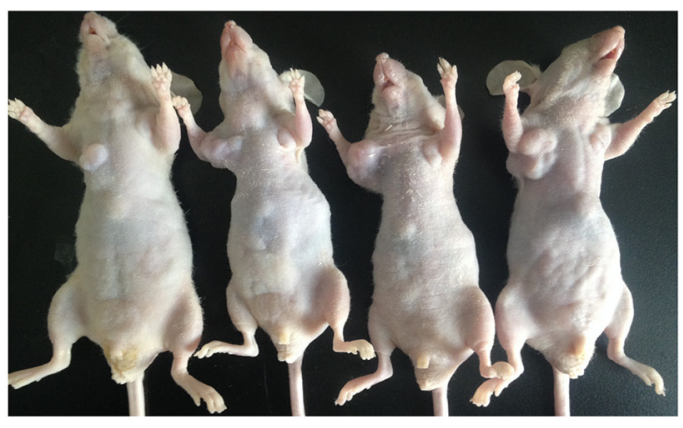

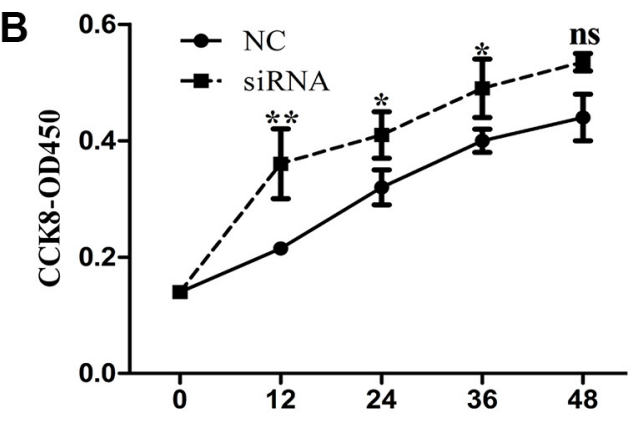
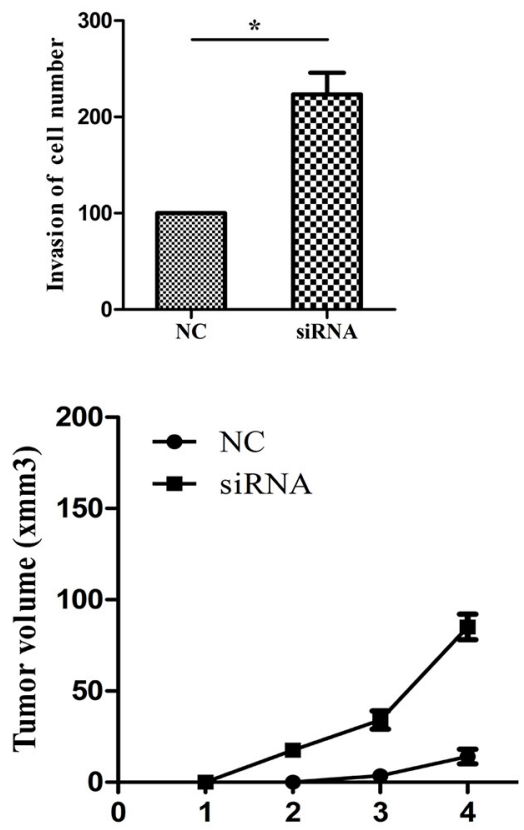
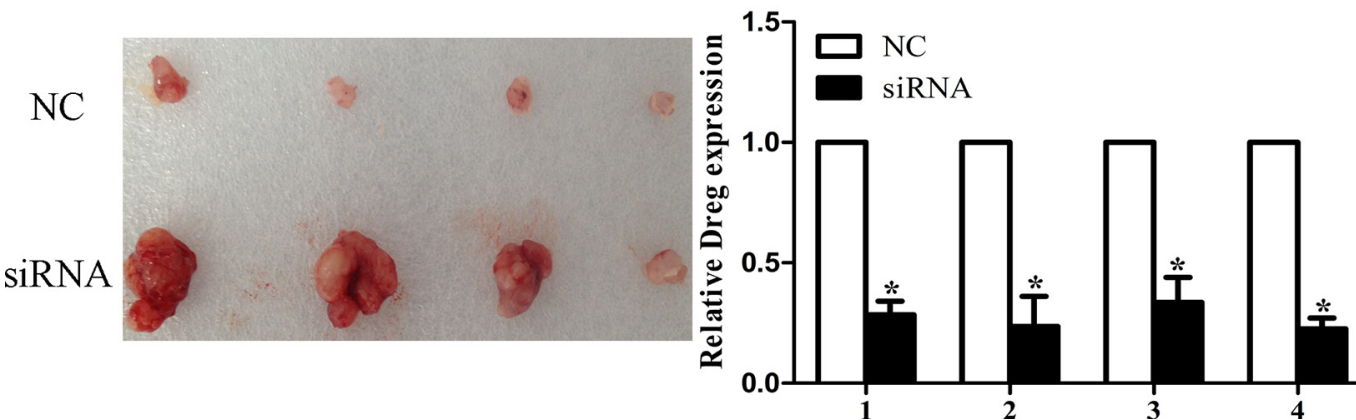

Figure 2: LncRNA AF147447 inhibition promote GC proliferation and invasion in vitro and in vivo. (A) LncRNA Af147447 expression level after AF147447 siRNA transfection was validated by qRT-PCR. (B) MKN45 cells were seeded in 96-well plates, and cell proliferation was assessed daily for 3 days using the Cell Counting Kit-8 (CCK-8) assay. (C) Cells were treated with either AF147447 siRNA or siRNA-NC for $24 \mathrm{~h}$. The representative images of invasive cells at the bottom of the membrane stained with crystal violet were visualized as shown. All experiments were performed in triplicate. (D) The in vivo models used were xenograft-transplanted nude mouse tumor models of human gastric cancer established with Af147447 siRNA or siRNA control. The left arms of the mice were injected with control GC cell and the right arms of the mice were injected with AF147447 siRNA GC cells. Photographs of tumors are presented. Effects of lncRNA AF147447 expression in nude mouse models are shown. $(* p<0.05, * * p<0.01)$. 
compared with those transfecting with the siRNA control. These results suggested that AF147447 could inhibit MUC2 RNA and protein expression.

We also determined MUC2 RNA and protein levels after cells infected with different density of $H$. pylori bacteria. After $H$. pylori infection, the infected cells showed markedly increased MUC2 RNA and protein expression (Figure 5A and 5B). Then, we detected its expression both in 75 pairs of $H$. pylori infected and noninfected tissues and 50 pairs of tumor tissues by qRTPCR. The results showed that MUC2 expression was markedly higher in infected gastric tissues, especially in infected tumor tissues (Figure 5C). Immunohistochemistry analysis also suggested that MUC2 expression was upregulated in the infected tissues (Figure 5C). We selected 15 tissues and detected AF147447 and MUC2 expression
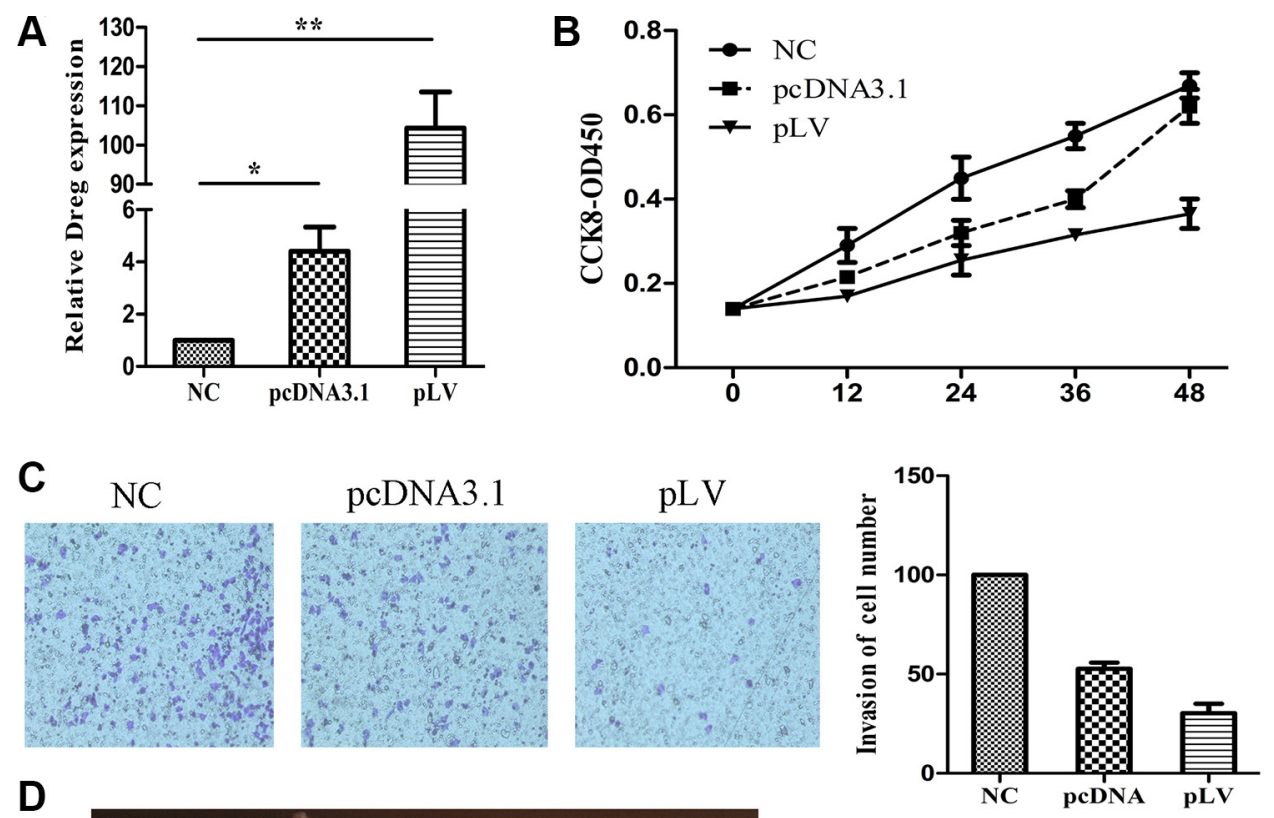

\section{D}
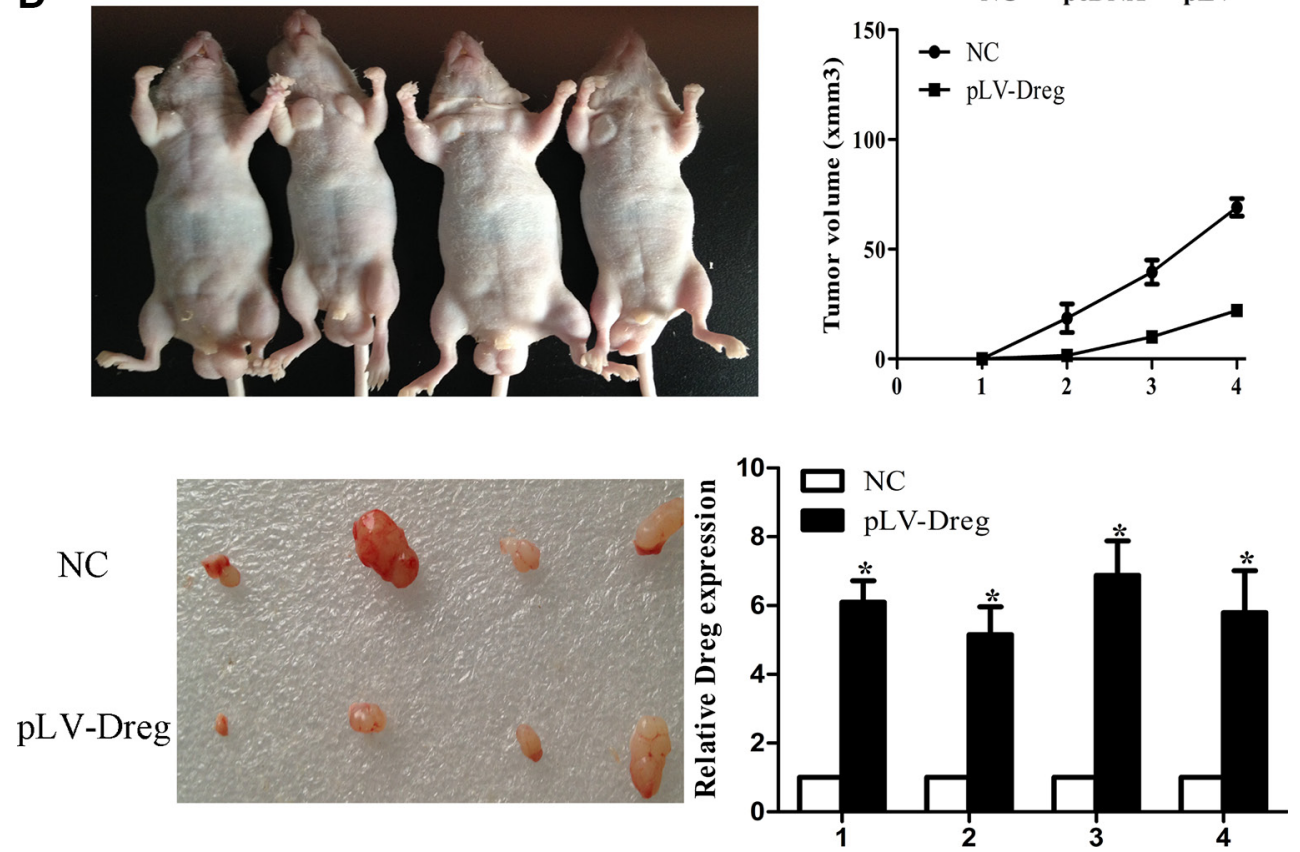

Figure 3: LncRNA AF147447 over-expression inhibit GC proliferation and invasion in vitro and in vivo. (A) Af147447 expression level after AF147447 over-expression was validated by qRT-PCR. (B) 7901 cells were seeded in 96-well plates, and cell proliferation was assessed daily for 3 days using the Cell Counting Kit-8 (CCK-8) assay. (C) Cells were treated with either pcDNAAF147447 or pLV-AF147447 for $24 \mathrm{~h}$. The representative images of invasive cells at the bottom of the membrane stained with crystal violet were visualized as shown. All experiments were performed in triplicate. (D) The in vivo models used were xenograft-transplanted nude mouse tumor models of human gastric cancer established with pLV-AF147447 or pLV-NC. The left arms of the mice were injected with pLV-AF147447 GC cells and the right arms of the mice were injected with pLV-NC cells. Photographs of tumors are presented. Effects of lncRNA AF147447 expression in nude mouse models are shown. $\left(* p<0.05,{ }^{*} p<0.01\right)$. 
respectively. From pearson correlation analysis, we could conclude that MUC2 expression was negatively correlated with AF147447 $(\mathrm{r}=-0.864, p=0.000)$ (Figure 5D).

\section{LncRNA AF147447 regulate miR-34c expression which could target MUC2}

We further investigated whether lncRNA AF147447 could alter miRNA expression and thus affect MUC2 expression. Firstly, we searched from miRanda database and found that miR-34c might be one regulatory gene of MUC2. We constructed luciferase gene and found that miR-34c mimic transfection significantly reduced the luciferase activity, which confirmed that MUC2 is miR-34c target gene (Figure 6A). By searching online databases, including PicTar, miRbase and BiBi Serv, the co-regulation of miRNA with lncRNA AF147447 was predicted by the bioinformatics analysis. The binding of miR-34c with lncRNA AF147447 was confirmed with the luciferase reporter assay (Figure 6B). We transfected with miR-34c mimics or inhibitor and found that MUC2 mRNA and protein (Figure 6C) were significantly decreased after transfecting with miR-34c mimics and increased after transfecting with inhibitor. We then co-transfected with

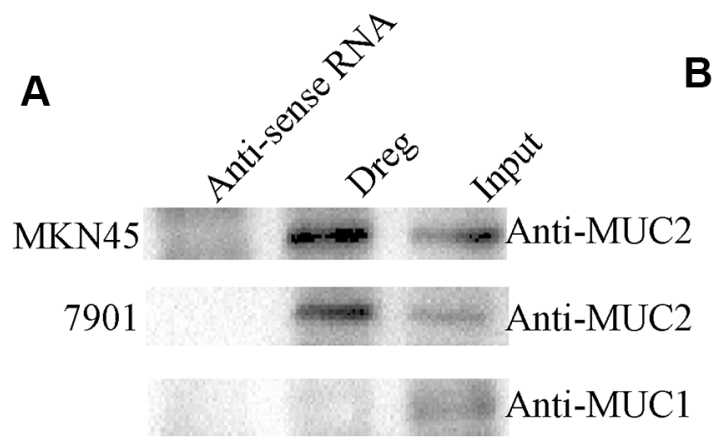

B

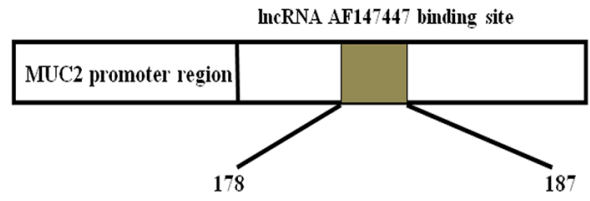

WT MUC2 promoter: 5' TCTGGCAGGACACCCCTCCCCCGGC3'

LncRNA AF 147447: 3' GGTGAGGAAGGGAGGAGGGGGCCC5

MUT MUC2 promoter: 5'TCTGGCAGGACACCAAGATATATAC3'

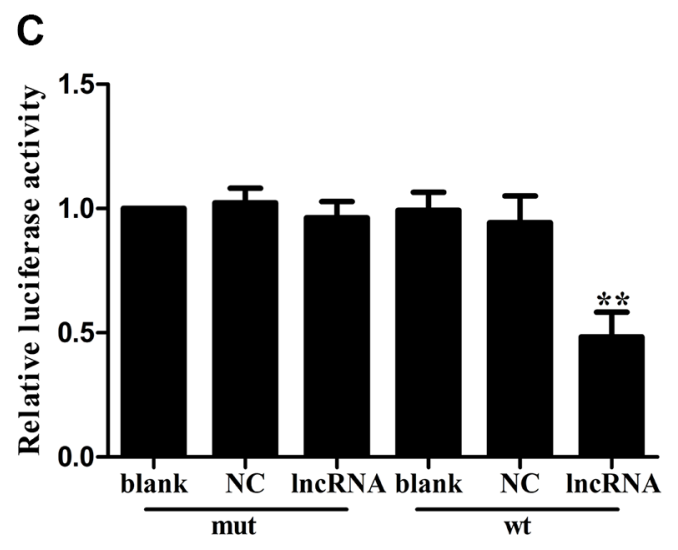

D
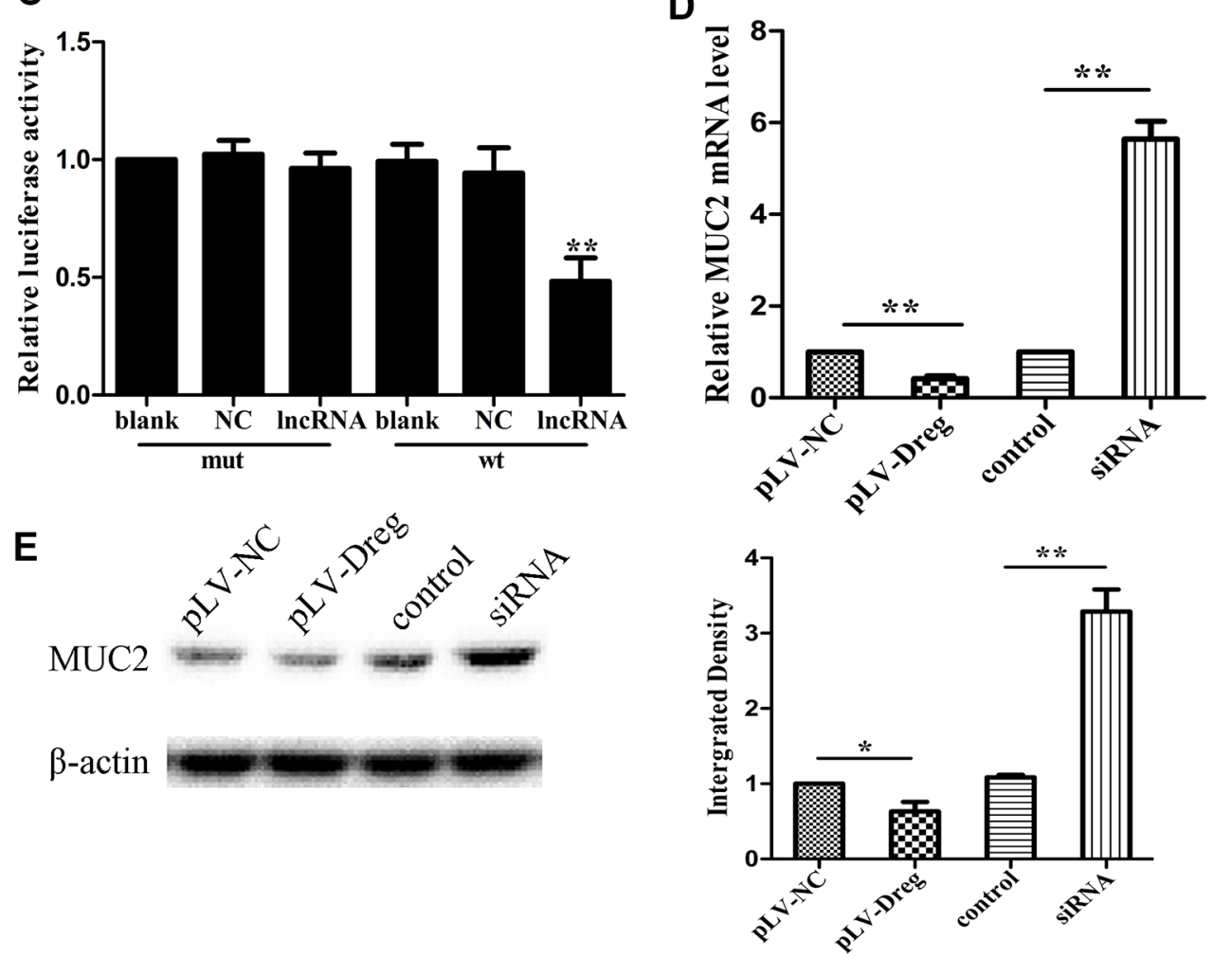

Figure 4: Identification and validation of IncRNA-AF147447 targets. (A) Biotinylated lncRNA-AF147447 or antisense RNA were incubated with nuclear extracts, targeted with streptavidin beads, and washed, and associated proteins were resolved in a gel. Western blotting analysis of the specific association of MUC2 and lncRNA-AF147447. Another MUC family: MUC1 is shown as a control. (B) Diagram of MUC2 promoter region constructs. (C) Luciferase reporter assays in 7901 cells, with co-transfection of wt or mt and lncRNA as indicated. (D) MUC2 expression was validated by qRT-PCR after transfecting with pLV-AF147447 or siRNA or their respective controls. (E) MUC2 expression was validated by western blot after transfecting with pLV-AF147447 or siRNA or their respective controls. $(* p<0.05 ; * p<0.01)$. 
mimic and si- AF147447 and found that the inhibition of MUC2 expression by miR-34c mimic was abrogated after si-AF147447 transfected. We also co-transfected with inhibitor and pLV-AF147447 and found that MUC2 overexpression was overcame after pLV-AF147447 transfected (Supplementary Figure S3A and S3B). We next examined the expression level of AF147447 after transfecting with miR-34c mimic or inhibitor. AF147447 was higher when miR-34c over-expressed and vice versa (Figure 6D). We also examined miR-34c expression after lncRNA-AF147447 overexpressed and down-regulated and found that miR-34c was significantly higher when lncRNA AF147447 overexpressed and vice versa (Figure $6 \mathrm{D})$. These results suggested that lncRNA-AF147447 and $\mathrm{miR}-34 \mathrm{c}$ was positively correlated in vitro. We also examined the correlation between miR-34c and Af147447 in vivo and found that they were also positively correlated (Figure 6D). Besides MUC2, EGFR and CD44 were also predicted to be miR-34c targets. We over-expressed or inhibit lncRNA-AF147447 and found that EGFR or CD44 was decreased after lncRNA AF147447 overexpressed and increased after lncRNA AF147447 inhibited
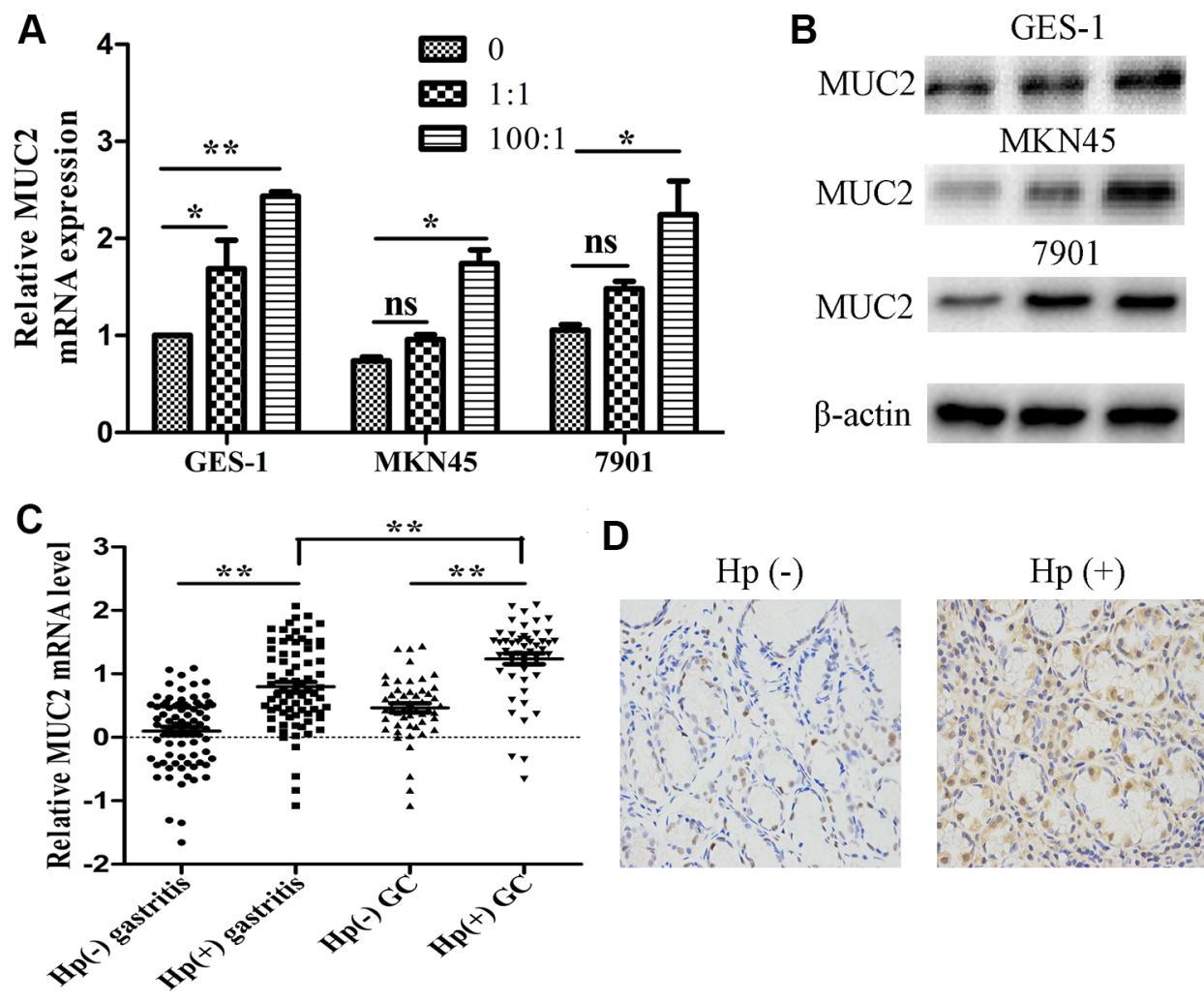

D

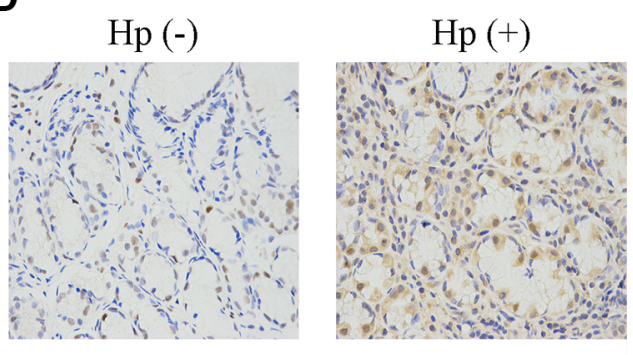

E
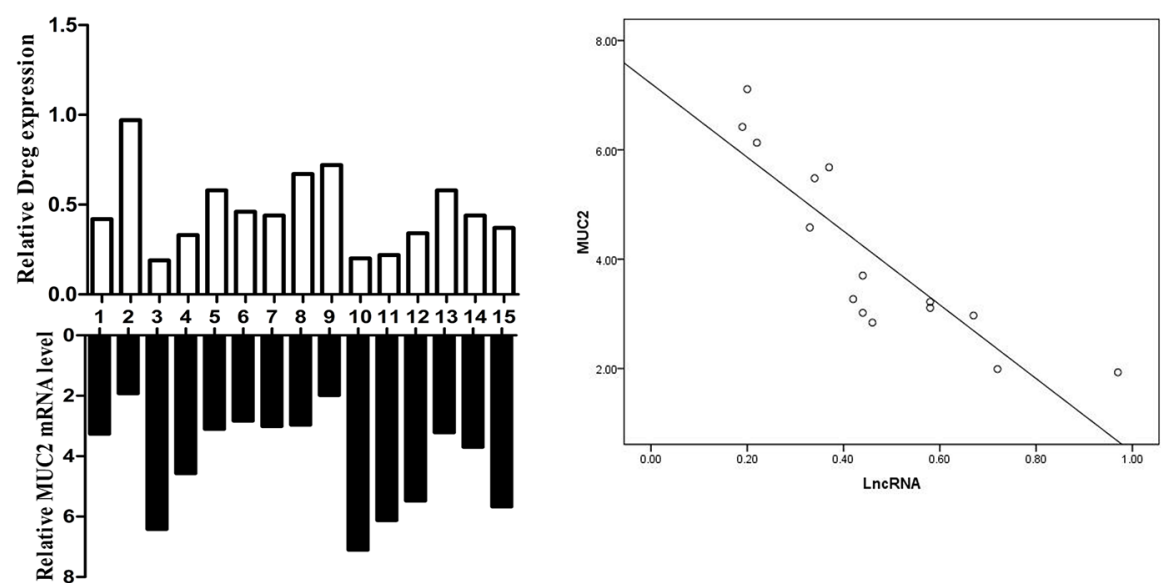

Figure 5: MUC2 expression under $\boldsymbol{H}$. pylori infected state. (A) MUC2 mRNA expression in $H$. pylori infected cells compared with controls. (B) MUC2 protein expression in H. pylori infected cells compared with controls. (C) MUC2 mRNA expression in H. pylori infected control and tumor tissues. (D) MUC2 protein expression in H. Pylori positive and negative tissues was detected by immunohistochemical staining. (E) A statistically significant inverse correlation between MUC2 and lncRNA-AF147447 levels in clinical specimens (Spearman's correlation analysis, $\mathrm{r}=-0.864, p=0.000)\left({ }^{*} p<0.05 ; * * p<0.01\right)$. 
(Supplementary Figure S3C and S3D). Taken together, these results demonstrated that 1 cRNA could regulate MUC2 expression not only by directing binding, but also through post-transcriptional pathway, such as regulating miRNA expression.

\section{H. pylori infection decreased IncRNA AF147447 expression by recruiting transcriptional factor E2F1}

A computational screen was conducted and predicted that transcriptional factor E2F1 was localized within AF147447 transcriptional element (Supplementary Figure S3). Recent studies have reported that transcription factors can bind to the promoter region of target lncRNAs and inhibit their expression. We wondered whether down-expression of AF147447 was mediated by this transcriptional factor. Firstly, to validate whether E2F1 could recruit to AF147447 promoter region and direct interaction of lncRNAAF147447 with E2F1, we performed RIP-qPCR and the results demonstrated that the lncRNA AF147447 RIP is significantly enriched for E2F1 compared to $\mathrm{IgG}$ (Figure 7A). The association between lncRNA AF147447 and E2F1 was further validated by luciferase
A

MUC2 3'UTR Wild Type
hsa-miR-34c
MUC2 3'UTR Mutant

B

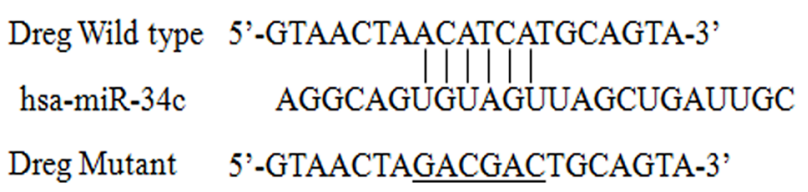

C
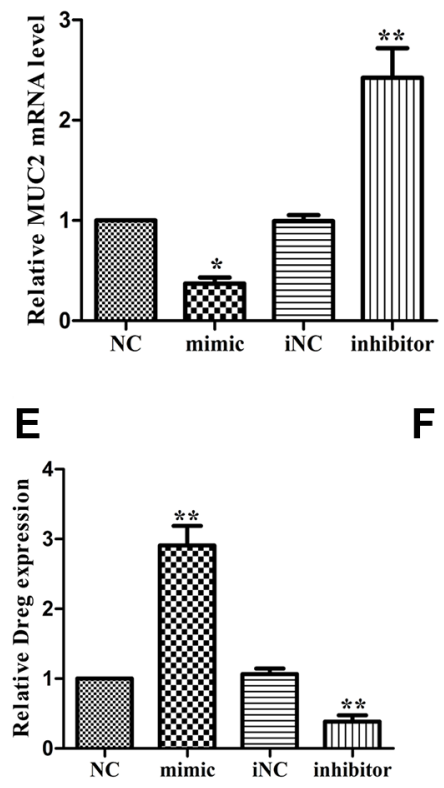

$\mathbf{F}$

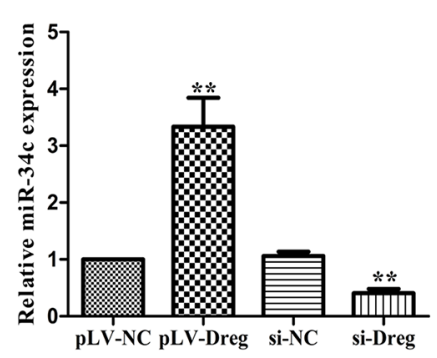

D

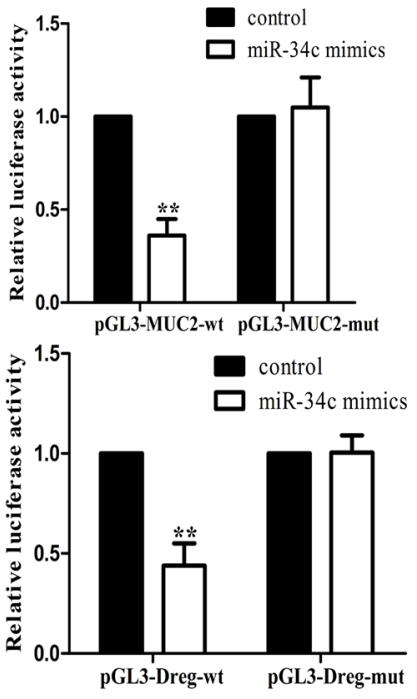

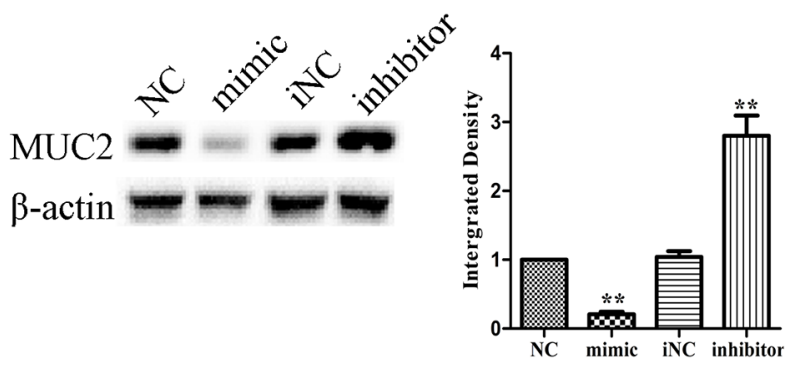

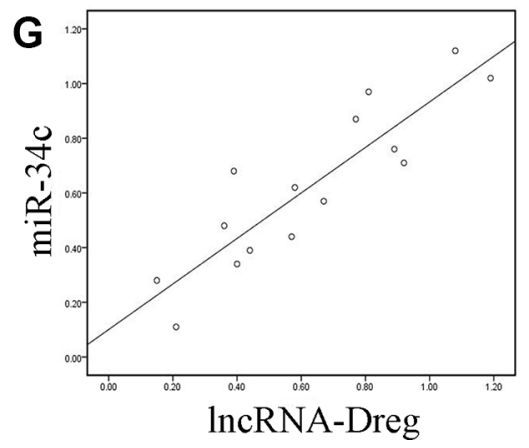

Figure 6: LncRNA-AF147447 interact with increased miR-34c expression level. (A) Diagram of MUC2 3'UTR region constructs. Luciferase reporter assays in 7901 cells, with co-transfection of miR-34c mimics and wt or mut 3'UTR. (B) Diagram of AF147447 wt and mut constructs. Luciferase reporter assays in 7901 cells, with co-transfection of miR-34c mimic and AF147447 wt or mut. (C) MUC2 mRNA expression was validated by qRT-PCR after cells transfecting with mimic or inhibitor. (D) MUC2 protein expression was validated by western blot after cells transfecting with mimic or inhibitor. (E) AF147447 expression was validated by qRT-PCR after cells transfecting with mimic or inhibitor. (F) MiR-34c expression was validated by qRT-PCR after cells transfecting pLV-AF147447 or si-AF147447. (G) Pearson analysis showed that miR-34c and lncRNA AF147447 was positively correlated. $\left({ }^{*} p<0.05 ; * * p<0.01\right)$. 
reporter analysis. We constructed AF147447 wt and mut promoter and found that lncRNA AF147447 transfection could significantly reduce the luciferase activity, which could be reversed by si-E2F1 co-transfection (Figure 7B). Next, we investigated the functional association between E2F1 and lncRNA AF147447. After transfecting with siE2F1, we found that lncRNA AF147447 was significantly over-expressed while transfecting with pcDNAE2F1, lncRNA AF147447 was significantly reduced (Figure 7C), which suggested that E2F1 could change
lncRNA AF147447 expression. We further examined whether E2F1 transfection could alter target gene MUC2 expression and found that MUC2 was significantly lower when si-E2F1 transfected and higher when pcDNA-E2F1 transfected (Figure 7D). We then validated the correlation between E2F1 expression in vivo with lncRNA AF147447 and MUC2. The results showed that E2F1 expression was negatively correlated with lncRNA AF147447 (Supplementary Figure S4A) and positively correlated with MUC2 (Supplementary Figure S4B).
A
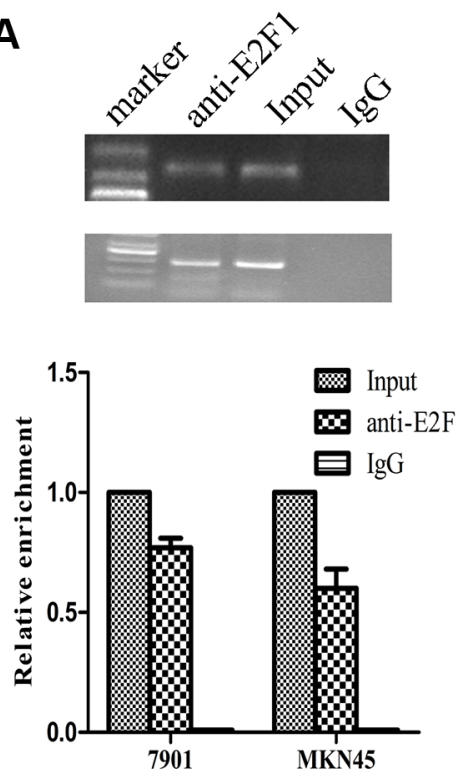

B

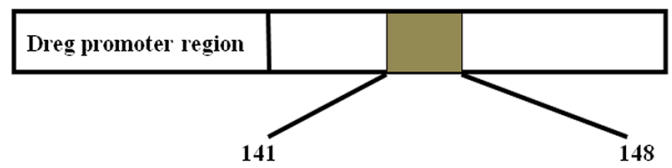

WT Dreg promoter: 5'TCTTCTTCCTTTCCCGGGAGGTGGGC3 E2F1: 3' AGGTTGAAGAAAGGGCAAGAAGGG5'

MUT Dreg promoter: 5'TCTTCTTCCGCGATATGGAGGTGGGC

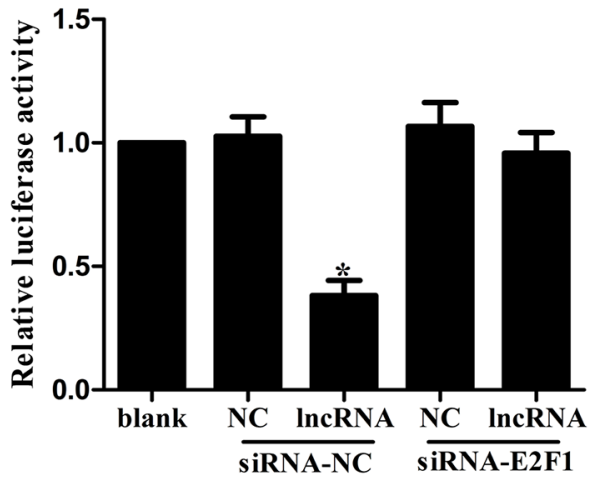

D

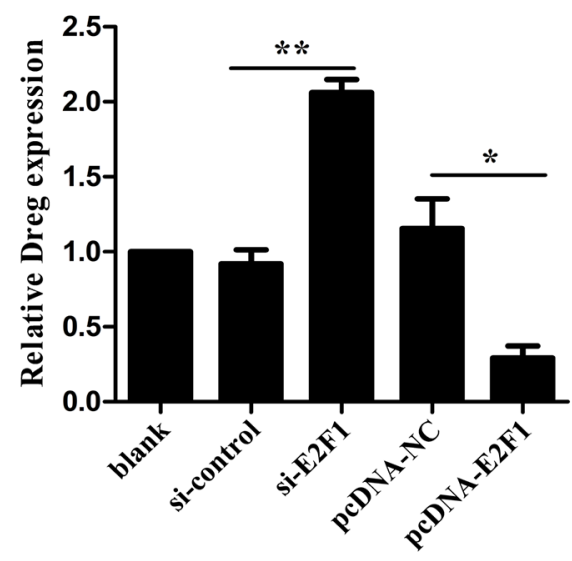

Figure 7: LncRNA AF147447 physically associates with E2F1. (A) RIP experiments were performed using the E2F1 antibody to immunoprecipitate (IP) and a primer to detect lncRNA-AF147447. RIP enrichment was determined as RNA associated with E2F1 IP relative to an input control. (B) Diagram of lncRNA-AF147447 promoter region constructs. Luciferase reporter assays in 7901 cells, with co-transfection of IncRNA-AF147447, with siRNA-E2F1 or siRNA-control. (C) LncRNA-AF147447 expression was detected by qRTPCR after cells transfecting with si-E2F1 or pcDNA-E2F1. (D) MUC2 mRNA and protein were detected by qRT-PCR and western blot. $\left({ }^{*} p<0.05 ; * * p<0.01\right)$. 


\section{DISCUSSION}

In this study, as shown in Supplementary Figure S4C, we reported that after $H$. pylori infection, IncRNA AF147447 expression level was decreased and its downregulation could inhibit GC cell proliferation and invasion by inducing target oncogene, MUC2. Therefore, IncRNA AF147447 plays an important role in H. pylori infectionrelated GC. We also found that lncRNA AF147447 was markedly decreased in GC and was further decreased in $H$. pylori positive GC. By applying gain-of-function and loss-of-function methods, lncRNA AF147447 was identified to significantly inhibit cell proliferation and invasion. Additionally, it was shown that the expression of lncRNA AF147447 was regulated by E2F1 and oncogene MUC2 was a direct target of AF147447. It can also been found that AF147447 could post-transcriptionally affect MUC2 expression by binding with miR-34c.

$\mathrm{GC}$ is a worldwide malignant disease with a high incidence, high recurrence and poor prognosis, especially in Southeast Asia [12]. Significant proportion of the GC patients was $H$. pylori infected. Consequently, comprehensively study the mechanism and then develop new targeted treatments for $H$. pylori infection related GC was indeed necessary. Epigenetic modification belongs to one of the many characteristics of early process of tumorigenesis, changing the surrounding microenvironment to form a tumor [13]. Protein coding and non-protein coding genes (microRNA, lncRNA, circRNA) dysregulation has been identified to be able to diagnosing and predicting GC patients' prognosis [14]. However, whether $H$. pylori infection could promote GC through lncRNA regulation has not been previously elucidated. In the present study, we found that patients with the severe histology had much lower lncRNA AF147447 expression than normal controls. Previous reports showed that deregulated lncRNAs in GC could also be used as prognostic and diagnostic biomarkers [15]. Detecting the expression level of these lncRNAs, in combination with lncRNA AF147447 and even protein-coding genes or miRNAs, may be valuable to predict the prognosis of $H$. pylori related patients more accurately [16]. Furthermore, like some other lncRNAs, for example M86523 identified in our microarray analysis, are also suggested to be differentially expressed in positive and negative tissues, we will next determine whether deregulation of these lncRNAs also correlates with the histology of the patients and whether detecting these lncRNAs together is more precise in identifying the prognosis of GC patients [16].

We further examined the role of AF147447 in vitro and we found that AF147447 could inhibit tumor proliferation and invasion. We then explored that GC cells with over-expression or down-regulation of AF147447 might play a critical role in tumor growth in vivo. Furthermore, an RNA pull-down assay syggested that AF147447 was able to combine with transcription factor
E2F1 and its expression level was mediated by E2F1. E2F1 was a transcription factor which over-expressed by $H$. pylori infection [17]. Transcription factor could combine with the promoter of the gene and suppress its expression [18]. We have also identified a target gene, MUC2, as affected by AF147447 down-regulation. The mucin core proteins (MUCs) are glycosylated proteins expressed in tissue-specific patterns in the gastrointestinal tract [19]. It has been found over-expressed in various cancers, such as colorectal cancer [20], ovarian cancer [21]. MUC2 over-expression is an adverse prognostic factor in cancers [22]. The regulatory role of MUC2 in tumor progression suggests that MUC2 could be considered as a new diagnostic marker for cancer diagnosis and a new drug target for the cancer therapy [23]. We also found that lncRNA AF147447 could regulate MUC2 expression by regulating miR-34c expression. From pearson correlation analysis, we found that miR$34 \mathrm{c}$ and lncRNA AF147447 was positively correlated in tissues. Besides, IncRNA AF147447 transfection could alter miR-34c expression and vice versa.

As some studies have shown that H. pylori infection could change the expression of certain kinds of lncRNA [24], our current study is one of the important supplements to the H. pylori related carcinogenesis, which provided a brand new lncRNA diagnostic biomarker and therapeutic target for GC. However, the roles of other differentially expressed lncRNAs identified from the microarray analysis need further validation and analysis.

\section{MATERIALS AND METHODS}

\section{Patient samples}

Seventy-five pairs of $H$. pylori positive and negative tissues were obtained from patients who underwent gastroscrope and 50 pairs of $H$. pylori positive and negative tumor tissues were obtained from patients who underwent gastrectomy in Jiangsu province Hospital. The basic characteristics of the enrolled patients were listed in Supplementary Table S1. For the use of materials for research purposes, written informed consent was obtained from each patient. The consent procedure and study protocol were approved by the Medical Institutional Ethical Committee of first affiliated hospital of Nanjing medical university.

\section{Microarray detection and bioinformatics analyses}

Three pairs of gastric tissues from $H$. pylori positive and negative patients were randomly selected for microarray analysis. Total RNA was extracted, amplified, and transcribed into fluorescent cDNA. Labeled samples were hybridized to the Human LncRNA Array. Differentially expressed lncRNAs with statistical 
significance were identified through Volcano Plot filtering. The threshold to screen up- or down-regulated lncRNAs was fold change $>2$ and $P<0.05$.

\section{Cell culture}

Human gastric cancer cell lines 7901 and MKN45 were obtained from American Type Culture Collection (ATCC). All cell lines were maintained in RPMI 1640 supplemented with $10 \%$ fetal calf serum at $37^{\circ} \mathrm{C}$ in a humidified atmosphere of $95 \%$ air and $5 \% \mathrm{CO}_{2}$. Cell lines have identified by authentication.

\section{Cell proliferation assay and transwell invasion assay}

Cell proliferation was assayed using CCK8 (Roche, Basel, Switzerland). Invasion was assessed using the in vitro assay, as described previously [22].

\section{In vivo assays for tumor growth}

Cells $\left(1 \times 10^{7}\right)$ that had been stably transfected with pLV-AF147447/pLV-NC or transient transfected with siRNA/control were suspended in $100 \mu \mathrm{L}$ PBS and implanted subcutaneously into the bilateral armpit of $\mathrm{BALB} / \mathrm{c}$ nude mice. The tumors were measured every three days after implantation, and the volume of each tumor was calculated by length $\times$ width $^{2} \times 0.5$.

\section{Quantitative real-time PCR}

Total RNA was extracted using Trizol reagent (TAKARA, Japan). First-strand cDNA was generated using the Reverse Transcription System Kit (TAKARA). Random primers were used for RT-PCR for IncRNAs since some lncRNAs do not have poly A tails. Quantitative real-time PCR was performed using a standard SYBR-Green PCR kit protocol on a StepOne System (Applied Biosystems, Foster, CA). GAPDH was used as an endogenous control. The primers were shown in Supplementary Table S3. qRT-PCR was performed using ABI Prism 7900HT (Applied Biosystems, Foster City, CA, USA) according to the direction of the reagents. The details are as described previously [22].

\section{Western blot analysis}

Total cell lysate was prepared in $1 \times$ SDS buffer. Equivalent amounts of proteins were separated by SDSPAGE and transferred onto NC membranes (Millipore, USA). After incubation with antibodies specific for either MUC2 (abcam, USA) or $\beta$-actin (Cell signaling Technology), blots were incubated with IRDye 800 antirabbit secondary antibodies. The integrated density of the band was quantified by the ImageJ software $(\mathrm{NIH}$, Bethesda, MD, USA).

\section{Immunohistochemical assay}

Sections were deparaffinized rinsed in PBS twice and incubated with $10 \%$ normal goat serum for $30 \mathrm{~min}$ to block non-specific antibody binding. After washing, samples were incubated with primary anti-rabbit antibody MUC2 (Abcam, USA) and then incubated with secondary antibodies. Then, the sections were stained with DAB according to the manufacturer's protocols and mounted and photographed using a digitalized microscope camera (Nikon, Tokyo, Japan).

\section{Rna pull-down assay}

LncRNA-AF147447 and its antisense RNA were in vitro transcribed from vector pLV-AF147447 and biotin-labeled with the Biotin RNA Labeling Mix (Roche Diagnostics, Indianapolis, IN) and T7/SP6 RNA polymerase, treated with RNase-free DNase I (Roche), and purified with an RNeasy Mini Kit (Qiagen, Valencia, CA). One milligram of protein from SGC-7901 cells stably transfected with pLV-AF147447 extracts was then mixed with 50 pmol of biotinylated RNA, incubated with streptavidin agarose beads (Invitrogen, Carlsbad, CA), and washed. The retrieved proteins were resolved by SDSPAGE) and the specific bands were excised and analyzed by mass spectrometry and western blot.

\section{RNA immunoprecipitation}

RIP experiments were performed using a Magna RIP RNA-Binding Protein Immunoprecipitation Kit (Millipore, Bedford, MA) according to the manufacturer's instructions. Antibody for RIP assays of E2F1 (Cell Signaling Technology, Beverly, MA) was diluted 1:1000. Coprecipitated RNAs were detected by RT-PCR. Gene specific primers used for detecting AF147447 are presented in Supplementary Table S3.

\section{Luciferase assay}

AF147447 or MUC2 promoter regions were amplified by PCR and cloned downstream of the firefly luciferase gene in pGL3 vector (Promega, USA). The vector was named wild-type (wt) promoter regions. Site-directed mutagenesis of the E2F1 or AF147447 binding site in the promoters was generated by Invitrogen and named mutant (mut) promoter regions. For reporter assays, wt or mut vector and the control vector pRL-CMV were co-transfected. Luciferase activity was measured 36 hours after transfection using the Dual-Luciferase Reporter Assay System (Promega).

\section{Statistical analysis}

Statistical analysis was performed using SPSS software (SPSS, Chicago, Illinois, USA). Comparisons between two groups were performed using Student's $t$-test 
or Mann-Whitney $U$ test, as appropriate. All values were expressed as mean $\pm \mathrm{SD}$. Two-tailed $t$ tests were applied to all data unless otherwise specified, with $P<0.05$ considered statistically significant.

\section{CONFLICTS OF INTEREST}

The authors declare that there are no conflicts of competing interest.

\section{FUNDING}

This work was supported by National Natural Science Foundation of China (No. 81270476 and 81470830) and the Priority Academic Program Development of Jiangsu Higher Education Institutions (JX10231801).

\section{REFERENCES}

1. Song JH, Meltzer SJ. MicroRNAs in pathogenesis, diagnosis, and treatment of gastroesophageal cancers. Gastroenterology. 2012; 143:35-47.e2.

2. Ohnita $\mathrm{K}$, Isomoto $\mathrm{H}$, Shikuwa $\mathrm{S}$, Yajima $\mathrm{H}$, Minami H, Matsushima K, Akazawa Y, Yamaguchi N, Fukuda E, Nishiyama H, Takeshima F, Nakao K. Early and long-term outcomes of endoscopic submucosal dissection for early gastric cancer in a large patient series. Exp Ther Med. 2014; 7:594-598.

3. Kupcinskas J, Wex T, Link A, Leja M, Bruzaite I, Steponaitiene R, Juzenas S, Gyvyte U, Ivanauskas A, Ancans G, Petrenkiene V, Skieceviciene J, Kupcinskas L, et al. Gene polymorphisms of micrornas in Helicobacter pylori-induced high risk atrophic gastritis and gastric cancer. PLoS One. 2014; 9:e87467.

4. Akhavan-Niaki H, Samadani AA. Molecular insight in gastric cancer induction: an overview of cancer stemness genes. Cell Biochem Biophys. 2014; 68:463-73.

5. Ørom UA, Shiekhattar R. Long noncoding RNAs usher in a new era in the biology of enhancers. Cell. 2013; 154:1190-3.

6. Morlando M, Ballarino M, Fatica A, Bozzoni I. The role of long noncoding RNAs in the epigenetic control of gene expression. ChemMedChem. 2014; 9:505-10.

7. Caputa G, Schaffer JE. RNA Regulation of Lipotoxicity and Metabolic Stress. Diabetes. 2016; 65:1816-23.

8. Boon RA, Jaé N, Holdt L, Dimmeler S. Long Noncoding RNAs: From Clinical Genetics to Therapeutic Targets? J Am Coll Cardiol. 2016; 67:1214-26.

9. Sánchez Y, Huarte M. Long non-coding RNAs: challenges for diagnosis and therapies. Nucleic Acid Ther. 2013; 23:15-20.

10. Zhang EB, Kong R, Yin DD, You LH, Sun M, Han L, Xu TP, Xia R, Yang JS, De W, Chen Jf. Long noncoding
RNA ANRIL indicates a poor prognosis of gastric cancer and promotes tumor growth by epigenetically silencing of miR-99a/miR-449a. Oncotarget. 2014; 5:2276-92. doi: 10.18632/oncotarget.1902.

11. Xu TP, Huang MD, Xia R, Liu XX, Sun M, Yin L, Chen WM, Han L, Zhang EB, Kong R, De W, Shu YQ. Decreased expression of the long non-coding RNA FENDRR is associated with poor prognosis in gastric cancer and FENDRR regulates gastric cancer cell metastasis by affecting fibronectin1 expression. J Hematol Oncol. 2014; 7:63.

12. Patru CL, Surlin V, Georgescu I, Patru E. Current issues in gastric cancer epidemiology. Rev Med Chir Soc Med Nat Iasi. 2013; 117:199-204.

13. Zhu X, Liu J, Xu X, Zhang C, Dai D. The Pleckstrin and Sec7 domain-containing gene as a novel epigenetic modification marker in human gastric cancer and its clinical significance. Int J Oncol. 2015; 46:195-204.

14. Osman MA, Bloom GS, Tagoe EA. Helicobacter pyloriinduced alteration of epithelial cell signaling and polarity: a possible mechanism of gastric carcinoma etiology and disparity. Cytoskeleton. 2013; 70:349-59.

15. Zhang E, Yin D, Han L, He X, Si X, Chen W, Xia R, Xu T, Gu D, De W, Guo R, Xu Z, Chen J. E2F1-induced upregulation of long noncoding RNA LINC00668 predicts a poor prognosis of gastric cancer and promotes cell proliferation through epigenetically silencing of CKIs. Oncotarget. 2016; 7:23212-26. doi: 10.18632/ oncotarget.6745.

16. Yang F, Zhang L, Huo XS, Yuan JH, Xu D, Yuan SX, Zhu N, Zhou WP, Yang GS, Wang YZ, Shang JL, Gao CF, Zhang FR, et al. Long noncoding RNA high expression in hepatocellular carcinoma facilitates tumor growth through enhancer of zeste homolog 2 in humans. Hepatology. 2011; 54:1679-89.

17. Kim JM, Kim JS, Yoo DY, Ko SH, Kim N, Kim H, Kim YJ. Stimulation of dendritic cells with Helicobacter pylori vacuolating cytotoxin negatively regulates their maturation via the restoration of E2F1. Clin Exp Immunol. 2011; 166:34-45.

18. Takashima Y, Guo G, Loos R, Nichols J, Ficz G, Krueger F, Oxley D, Santos F, Clarke J, Mansfield W, Reik W, Bertone P, Smith A. Resetting transcription factor control circuitry toward ground-state pluripotency in human. Cell. 2014; 158:1254-69.

19. Yonezawa S, Higashi M, Yamada N, Yokoyama S, Kitamoto S, Kitajima S, Goto M. Mucins in human neoplasms: clinical pathology, gene expression and diagnostic application. Pathol Int. 2011; 61:697-716.

20. Walsh MD, Clendenning M, Williamson E, Pearson SA, Walters RJ, Nagler B, Packenas D, Win AK, Hopper JL, Jenkins MA, Haydon AM, Rosty C, English DR, et al. Expression of MUC2, MUC5AC, MUC5B, and MUC6 mucins in colorectal cancers and their association with 
the $\mathrm{CpG}$ island methylator phenotype. Mod Pathol. 2013; 26:1642-56.

21. He YF, Zhang MY, Wu X, Sun XJ, Xu T, He QZ, Di W. High MUC2 expression in ovarian cancer is inversely associated with the M1/M2 ratio of tumor-associated macrophages and patient survival time. PLoS One. 2013; 8:e79769.

22. Kim DH, Shin N, Kim GH, Song GA, Jeon TY, Kim DH, Lauwers GY, Park DY. Mucin expression in gastric cancer: reappraisal of its clinicopathologic and prognostic significance. Arch Pathol Lab Med. 2013; 137:1047-53.
23. Yuan JH, Yang F, Wang F, Ma JZ, Guo YJ, Tao QF, Liu F, Pan W, Wang TT, Zhou CC, Wang SB, Wang YZ, Yang $Y$, et al. A long noncoding RNA activated by TGF- $\beta$ promotes the invasion-metastasis cascade in hepatocellular carcinoma. Cancer Cell. 2014; 25:666-81.

24. Yao Y, Jiang Q, Jiang L, Wu J, Zhang Q, Wang J, Feng H, Zang P. Lnc-SGK1 induced by Helicobacter pylori infection and highsalt diet promote Th2 and Th17 differentiation in human gastric cancer by SGK1/Jun B signaling. Oncotarget. 2016; 7:20549-60. doi: 10.18632/oncotarget.7823. 